\title{
Article \\ The Method of Fundamental Solutions for the 3D Laplace Inverse Geometric Problem on an Annular Domain
}

\author{
Mojtaba Sajjadmanesh ${ }^{1}$, Hassen Aydi ${ }^{2,3,4, *}$, Eskandar Ameer ${ }^{5}$ and Choonkil Park ${ }^{6, *}$ \\ 1 Faculty of Basic Science, University of Bonab, Bonab P.O. Box 55517-61167, Iran; m.sajjadmanesh@ubonab.ac.ir \\ 2 Institut Supérieur d'Informatique et des Techniques de Communication, Université de Sousse, \\ Sousse 4000, Tunisia \\ 3 China Medical University Hospital, China Medical University, Taichung 40402, Taiwan \\ 4 Department of Mathematics and Applied Mathematics, Sefako Makgatho Health Sciences University, \\ Ga-Rankuwa 0204, South Africa \\ 5 Department of Mathematics, Taiz University, Taiz 6803, Yemen; eskanderameer@taiz.edu.ye \\ 6 Research Institute for Natural Sciences, Hanyang University, Seoul 04763, Korea \\ * Correspondence: hassen.aydi@isima.rnu.tn (H.A.); baak@hanyang.ac.kr (C.P.)
}

check for updates

Citation: Sajjadmanesh, M.; Aydi, H.; Ameer, E.; Park, C. The Method of Fundamental Solutions for the 3D Laplace Inverse Geometric Problem on an Annular Domain. Fractal Fract. 2022, 6, 66. https://doi.org/ $10.3390 /$ fractalfract6020066

Academic Editor: Savin Treanță

Received: 11 December 2021

Accepted: 9 January 2022

Published: 27 January 2022

Publisher's Note: MDPI stays neutral with regard to jurisdictional claims in published maps and institutional affiliations.

Copyright: (C) 2022 by the authors. Licensee MDPI, Basel, Switzerland. This article is an open access article distributed under the terms and conditions of the Creative Commons Attribution (CC BY) license (https:// creativecommons.org/licenses/by/ $4.0 /)$.

\begin{abstract}
In this paper, we are interested in an inverse geometric problem for the three-dimensional Laplace equation to recover an inner boundary of an annular domain. This work is based on the method of fundamental solutions (MFS) by imposing the boundary Cauchy data in a leastsquare sense and minimisation of the objective function. This approach can also be considered with noisy boundary Cauchy data. The simplicity and efficiency of this method is illustrated in several numerical examples.
\end{abstract}

Keywords: inverse geometric problem; Laplace equation; method of fundamental solution; leastsquare problem

\section{Introduction}

The inverse geometry problems, as an important subclass of inverse problems, can be subdivided into two subclasses, depending on the location of the unknown boundary. In the first kind, the portion of the outer boundary of the solution domain is unknown, whilst in the second kind, the inner boundary is unknown.

There are many methods for solving the inverse geometry problems, such as the boundary element regularisation method by Lesnic et al. [1], the method of fundamental solutions and moving pseudo-boundary method by Karageorghis et al. [2-4], the boundary function method by Wang et al. [5], the conjugate gradient method (CGM) and the boundary element technique by Huang et al. [6,7].

Bin-Mohsin and Lesnic in 2012 utilised the method of fundamental solutions (MFS) to the modified Helmholtz inverse geometry problem on an annular domain [8].

The purpose of this paper is to extend the aforementioned current approach to the threedimensional Laplace equation based on the method of fundamental solutions. Finally, two examples are presented to show the simplicity and efficiency of this method.

\section{Formulation of the Inverse Geometric Problem}

Let $D \subset \mathbb{R}^{3}$ be a simply connected domain with an unknown boundary $\partial D$ which is compactly contained in a simply connected domain $\Omega \subset \mathbb{R}^{3}$ with the boundary $\partial \Omega$.

Let us consider the following inverse problem:

$$
\Delta u=0 \text { in } \Omega \backslash \bar{D} \text {, }
$$


subject to the boundary conditions,

$$
\begin{aligned}
& u=f \quad \text { on } \quad \partial \Omega, \\
& \frac{\partial u}{\partial n}=g \text { on } \partial \Omega, \\
& u=h \quad \text { on } \partial D,
\end{aligned}
$$

where $f \in H^{1 / 2}(\partial \Omega)$ and $g \in H^{-1 / 2}(\partial \Omega)$ are given functions and $n$ is an outward unit normal vector on $\partial \Omega$. Moreover, the function $h \in H^{1 / 2}(\partial D)$ is given on the unknown boundary $\partial D$. Without loss of generality, we can suppose that $\Omega$ is the unit disk $B(\underline{0} ; 1)$; otherwise we can conformally map the exterior of the simply connected domain $\Omega$ onto the exterior of the unit disk.

The unknown boundary $\partial D$ can be expressed in spherical coordinates as

$$
\partial D=\{r(\theta, \varphi)(\cos \theta \sin \varphi, \sin \theta \sin \varphi, \cos \varphi) ; \theta \in[0,2 \pi), \varphi \in[0, \pi]\}
$$

where $r(\theta, \varphi)$ is a $2 \pi$-periodic and $\pi$-periodic smooth function with respect to $\theta$ and $\varphi$, respectively, with values in the interval $(0,1)$.

The inverse problem we are concerned with is to determine geometrically the domain boundary $\partial D$ by utilising the method of fundamental solutions.

\section{The Least-Square Problem Based on the MFS}

In the classic MFS, the solution of a homogeneous linear partial differential equation (PDE) is approximated by a linear combination of the fundamental solutions with the set of sources located outside the problem domain and a set of points on the domain boundary. The linear combination coefficients are determined by collocation or, alternatively, with a least-squares fit of the boundary conditions.

Based on the MFS, one can approximate the solution of (1) by a linear combination of its fundamental solutions, which is given by [9]

$$
U(\mathbf{z}, \mathbf{s})=\frac{1}{4 \pi r} ; r=\|\mathbf{z}-\mathbf{s}\|
$$

i.e.,

$$
u(\mathbf{z})=\sum_{j=1}^{n_{s}} c_{j} U\left(\mathbf{z}, \mathbf{s}_{j}\right),
$$

where the collocation points $\mathbf{z}_{i}$ and $\mathbf{z}_{i+M}$ are uniformly located on $\partial \Omega$ and $\partial D$, respectively, i.e.,

$$
\begin{aligned}
& \mathbf{z}_{i}=\left(\cos \hat{\theta}_{i} \sin \hat{\varphi}_{i}, \sin \hat{\theta}_{i} \sin \hat{\varphi}_{i}, \cos \hat{\varphi}_{i}\right), \quad i=\overline{1, M} \\
& \mathbf{z}_{i+M}=r_{i}\left(\cos \theta_{i} \sin \varphi_{i}, \sin \theta_{i} \sin \varphi_{i}, \cos \varphi_{i}\right), \quad i=\overline{1, N}
\end{aligned}
$$

Further, the $n_{s}:=M+N$ source points $\mathbf{s}_{j}$ and $\mathbf{s}_{j+M}$ are uniformly located on the outside of $\bar{\Omega}$ and the inside of $D$, respectively, i.e.,

$$
\begin{aligned}
& \mathbf{s}_{j}=R_{1}\left(\cos \hat{\theta}_{j} \sin \hat{\varphi}_{j}, \sin \hat{\theta}_{j} \sin \hat{\varphi}_{j}, \cos \hat{\varphi}_{j}\right), \quad j=\overline{1, M} \\
& \mathbf{s}_{j+M}=\frac{r_{j}}{R_{2}}\left(\cos \theta_{j} \sin \varphi_{j}, \sin \theta_{j} \sin \varphi_{j}, \cos \varphi_{j}\right), \quad j=\overline{1, N}
\end{aligned}
$$

where $R_{1}, R_{2}>1$. 
The coefficients vector $\mathbf{c}=\left(c_{j}\right)_{j=\overline{1, M+N}}$ in linear combination (6) and also, the radial vector $\mathbf{r}=\left(r_{j}\right)_{j=\overline{1, N}}$ can be determined by imposing the boundary conditions (2)-(4) in a least-square sense, which recasts into minimising the objective function

$$
T(\mathbf{c}, \mathbf{r})=\|u-f\|_{L^{2}(\partial \Omega)}^{2}+\left\|\frac{\partial u}{\partial n}-g\right\|_{L^{2}(\partial \Omega)}^{2}+\|u-h\|_{L^{2}(\partial D)}^{2} .
$$

Upon discretisation, Equation (11) yields

$$
\begin{aligned}
T(\mathbf{c}, \mathbf{r}) & =\sum_{i=1}^{M}\left[\sum_{j=1}^{M+N} c_{j} U\left(\mathbf{z}_{i}, \mathbf{s}_{j}\right)-f\left(\mathbf{z}_{i}\right)\right]^{2}+\sum_{i=M+1}^{2 M}\left[\sum_{j=1}^{M+N} c_{j} \frac{\partial U}{\partial n}\left(\mathbf{z}_{i-M}, \mathbf{s}_{j}\right)-g\left(\mathbf{z}_{i-M}\right)\right]^{2} \\
& +\sum_{i=2 M+1}^{2 M+N}\left[\sum_{j=1}^{M+N} c_{j} U\left(\mathbf{z}_{i-M}, \mathbf{s}_{j}\right)-h\left(\mathbf{z}_{i-M}\right)\right]^{2} .
\end{aligned}
$$

In general, the boundary data $F \in\{f, g, h\}$ are measured noisy data satisfying

$$
F_{i}^{\delta}=F_{i}+\delta \operatorname{rand}(i) F_{i}
$$

where $\delta$ is the percentage noise and the number rand $(i)$ is a random number drawn from the standard uniform distribution on the interval $[-1,1]$ generated by the MATLAB code $-1+2 \operatorname{rand}(i)$.

Imposing noise on all measured data implies

$$
\begin{aligned}
T^{\delta}(\mathbf{c}, \mathbf{r}) & =\sum_{i=1}^{M}\left[\sum_{j=1}^{M+N} c_{j} U\left(\mathbf{z}_{i}, \mathbf{s}_{j}\right)-f^{\delta}\left(\mathbf{z}_{i}\right)\right]^{2}+\sum_{i=M+1}^{2 M}\left[\sum_{j=1}^{M+N} c_{j} \frac{\partial U}{\partial n}\left(\mathbf{z}_{i-M}, \mathbf{s}_{j}\right)-g^{\delta}\left(\mathbf{z}_{i-M}\right)\right]^{2} \\
& +\sum_{i=2 M+1}^{2 M+N}\left[\sum_{j=1}^{M+N} c_{j} U\left(\mathbf{z}_{i-M}, \mathbf{s}_{j}\right)-h^{\delta}\left(\mathbf{z}_{i-M}\right)\right]^{2} \cdot
\end{aligned}
$$

The minimisation of (12) or (14) imposes $2 M+N$ nonlinear equations for the $2 N+M$ unknowns $(\mathbf{c}, \mathbf{r})$, and for a unique solution, it is necessary that $M \geq N$.

\section{Error Analysis and the Regularisation}

The accuracy of the presented method is evaluated by the normalised relative root mean square error (RMSE) and $L_{\infty}$-error:

$$
\mathrm{RMSE}=\frac{\left\{\frac{1}{N} \sum_{i=1}^{N}\left|r_{i}^{(\text {an })}-r_{i}^{(\text {num })}\right|^{2}\right\}^{\frac{1}{2}}}{\max _{1 \leq i \leq N}\left|r_{i}^{(a n)}\right|}, L_{\infty} \text {-error }=\max _{1 \leq i \leq N}\left|r_{i}^{(a n)}-r_{i}^{(\text {num })}\right|
$$

where $r_{i}^{(\text {an })}$ and $r_{i}^{(\text {num })}$ denote the analytical and numerical radial vectors, respectively, at the $i^{\text {th }}$ collocation point on the boundary $\partial D$.

The obtained numerical radial vectors from the presented method are unstable, especially when noise is added to the boundary data, and so the regularisation is needed. For this, we can add the following regularisation terms via standard zeroth- and first-order Tikhonov's regularisation with parameters $\lambda_{1}, \lambda_{2} \geq 0$ to the functional (14), i.e.,

$$
\operatorname{Reg}(\underline{a}, \underline{r})=\sum_{j=2 M+N+1}^{3 M+2 N}\left(\sqrt{\lambda_{1}} a_{j-2 M-N}\right)^{2}+\sum_{j=3 M+2 N+1}^{3 M+3 N-1}\left(\sqrt{\lambda_{2}}\left(r_{j-3 M-2 N+1}-r_{j-3 M-2 N}\right)\right)^{2},
$$




\section{Numerical Examples}

In this section, we give some examples to check the effectiveness of the presented method. We consider a three-dimensional annular domain with an outer boundary as the unit sphere $\partial \Omega=B(\underline{0} ; 1), R_{1}=R_{2}=2$ and $M=N \in\{25,50\}$ in (7)-(10). Moreover, the percentage noise $\delta=5 \%$ is added to every measured boundary data.

The minimisation of functional (12) or (14) is carried out using the MATLAB optimisation toolbox routine lsqnonlin, which solves nonlinear least-squares problems.

Example 1. Consider a three-dimensional annular domain with an unknown inner boundary $\partial D=B\left(\underline{0} ; r^{(a n)}\right)$ of radius $r^{(a n)}=0.7$. The boundary data are given as follows:

$$
\begin{aligned}
& \left.u\right|_{\partial \Omega}=f(\theta, \varphi)=\frac{1}{2}\left\{\sin 2 \varphi(\cos \theta+\sin \theta)+\sin 2 \theta \sin ^{2} \varphi\right\}, \\
& \left.\frac{\partial u}{\partial n}\right|_{\partial \Omega}=g(\theta, \varphi)=\sin 2 \varphi(\cos \theta+\sin \theta)+\sin 2 \theta \sin ^{2} \varphi, \\
& \left.u\right|_{\partial D}=h(\theta, \varphi)=\frac{49}{200}\left\{\sin 2 \varphi(\cos \theta+\sin \theta)+\sin 2 \theta \sin ^{2} \varphi\right\} .
\end{aligned}
$$

The exact solution for these input boundary data is $u(x)=x_{1} x_{2}+x_{1} x_{3}+x_{2} x_{3}$.

Table 1 gives the values of the objective functions and the corresponding errors obtained using the optimal initial guess $r_{0}$ and $M=N \in\{25,50\}$ without using regularisation parameters. It can be seen that the values of the corresponding errors increase with the number of collocation points and so regularisation is needed.

Table 1. The values of the optimal initial guess, $r_{0}$, objective functions and the corresponding errors with $M=N \in\{25,50\}$ and no regularisation parameters for Example 1 .

\begin{tabular}{cccccc}
\hline & $M=N$ & $r_{0}$ & Objective Functions & $L_{\infty}$-Error & RMSE \\
\hline \multirow{2}{*}{ Without noise } & 25 & $\underline{0.5}$ & $4.9013 \times 10^{-3}$ & 4.3884 & 1.4418 \\
& 50 & $\underline{0.6}$ & 6.1682 & 5.0114 & 1.7985 \\
\hline \multirow{2}{*}{ With noise 5\% } & 25 & $\underline{0.6}$ & 2.1799 & 2.1210 & $7.1903 \times 10^{-1}$ \\
& 50 & $\underline{0.6}$ & $1.8326 \times 10^{1}$ & 1.0113 & $9.1243 \times 10^{-1}$ \\
\hline
\end{tabular}

In Tables 2 and 3, we present the values of the objective functions and the corresponding errors with initial guess, $r_{0}$, obtained using the regularisation parameters $\lambda_{1}, \lambda_{2} \in\left\{0,10^{-6}, 10^{-3}, 10^{-1}\right\}$ with $M=N \in\{25,50\}$ and so, in Table 4, we give the minimal objective functions and the corresponding errors with initial guess $r_{0}$.

\begin{tabular}{|c|c|c|c|c|c|c|}
\hline & & & $r_{0}$ & Objective Functions & $L_{\infty}$-Error & RMSE \\
\hline \multirow{2}{*}{ 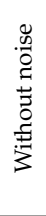 } & $\lambda_{1}=0$ & $\begin{array}{l}\lambda_{2}=10^{-6} \\
\lambda_{2}=10^{-3} \\
\lambda_{2}=10^{-1}\end{array}$ & $\begin{array}{l}\underline{0.7} \\
\underline{0.5} \\
\underline{0.3} \\
\end{array}$ & $\begin{array}{l}1.8102 \times 10^{-2} \\
2.5783 \times 10^{-3} \\
2.2118 \times 10^{-3}\end{array}$ & $\begin{array}{c}2.6100 \\
1.2932 \times 10^{-1} \\
1.0442 \times 10^{-1} \\
\end{array}$ & $\begin{array}{c}1.0249 \\
1.3835 \times 10^{-1} \\
1.3354 \times 10^{-1} \\
\end{array}$ \\
\hline & $\begin{array}{l}\lambda_{1}=10^{-6} \\
\lambda_{1}=10^{-3} \\
\lambda_{1}=10^{-1}\end{array}$ & $\lambda_{2}=0$ & $\begin{array}{l}\frac{0.6}{0.5} \\
\underline{0.7}\end{array}$ & $\begin{array}{c}5.8822 \times 10^{-3} \\
1.2470 \\
5.8688\end{array}$ & $\begin{array}{c}2.9436 \times 10^{-1} \\
2.8593 \\
5.1408 \times 10^{3}\end{array}$ & $\begin{array}{l}1.5451 \times 10^{-1} \\
8.8216 \times 10^{-1} \\
1.4688 \times 10^{-3}\end{array}$ \\
\hline \multirow{2}{*}{ 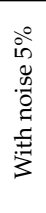 } & $\lambda_{1}=0$ & $\begin{array}{l}\lambda_{2}=10^{-6} \\
\lambda_{2}=10^{-3} \\
\lambda_{2}=10^{-1}\end{array}$ & $\begin{array}{l}\underline{0.9} \\
\underline{0.6} \\
\underline{0.5} \\
\end{array}$ & $\begin{array}{l}4.1621 \times 10^{-2} \\
1.3166 \times 10^{-2} \\
1.2386 \times 10^{-2} \\
\end{array}$ & $\begin{array}{l}3.5650 \times 10^{-1} \\
1.6224 \times 10^{-1} \\
1.0866 \times 10^{-1} \\
\end{array}$ & $\begin{array}{l}1.1951 \times 10^{-1} \\
1.3880 \times 10^{-1} \\
1.1611 \times 10^{-1}\end{array}$ \\
\hline & $\begin{array}{l}\lambda_{1}=10^{-6} \\
\lambda_{1}=10^{-3} \\
\lambda_{1}=10^{-1}\end{array}$ & $\lambda_{2}=0$ & $\begin{array}{l}\frac{0.4}{0.2} \\
\underline{0.2}\end{array}$ & $\begin{array}{c}3.1242 \times 10^{-2} \\
1.2775 \\
5.9897\end{array}$ & $\begin{array}{c}6.5845 \times 10^{-1} \\
8.0410 \times 10^{-1} \\
3.3646\end{array}$ & $\begin{array}{c}2.8637 \times 10^{-1} \\
3.8164 \times 10^{-1} \\
2.0861\end{array}$ \\
\hline
\end{tabular}

Table 2. The values of the optimal initial guess, $r_{0}$, objective functions and the corresponding errors using the regularisation parameters $\lambda_{1}, \lambda_{2}$ with $M=N=25$ for Example 1 . 
Table 3. The values of the optimal initial guess, $r_{0}$, objective functions and the corresponding errors using the regularisation parameters $\lambda_{1}, \lambda_{2}$ with $M=N=50$ for Example 1 .

\begin{tabular}{|c|c|c|c|c|c|c|}
\hline & & & $r_{0}$ & Objective Functions & $L_{\infty}$-Error & RMSE \\
\hline \multirow{2}{*}{ 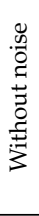 } & $\lambda_{1}=0$ & $\begin{array}{l}\lambda_{2}=10^{-6} \\
\lambda_{2}=10^{-3} \\
\lambda_{2}=10^{-1}\end{array}$ & $\begin{array}{l}\frac{0.6}{0.7} \\
\underline{0.7} \\
\end{array}$ & $\begin{array}{c}3.4092 \\
2.4650 \\
1.6998 \times 10^{-5} \\
\end{array}$ & $\begin{array}{c}4.6998 \\
8.8865 \times 10^{-1} \\
9.8441 \times 10^{-3}\end{array}$ & $\begin{array}{c}1.726 \\
4.5447 \times 10^{-1} \\
1.1249 \times 10^{-2} \\
\end{array}$ \\
\hline & $\begin{array}{l}\lambda_{1}=10^{-6} \\
\lambda_{1}=10^{-3} \\
\lambda_{1}=10^{-1}\end{array}$ & $\lambda_{2}=0$ & $\begin{array}{l}\underline{0.6} \\
\underline{0.5} \\
\end{array}$ & $\begin{array}{c}4.3927 \times 10^{-2} \\
9.7102 \times 10^{-1} \\
1.4145 \times 10^{1}\end{array}$ & $\begin{array}{l}4.7363 \\
4.6546 \\
1.2983\end{array}$ & $\begin{array}{l}1.8483 \\
1.9311 \\
1.5821\end{array}$ \\
\hline \multirow{2}{*}{ 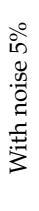 } & $\lambda_{1}=0$ & $\begin{array}{l}\lambda_{2}=10^{-6} \\
\lambda_{2}=10^{-3} \\
\lambda_{2}=10^{-1}\end{array}$ & $\begin{array}{l}\underline{0.6} \\
\underline{0.8} \\
\underline{0.6}\end{array}$ & $\begin{array}{c}1.2600 \\
1.2761 \times 10^{-2} \\
6.2499 \times 10^{-3}\end{array}$ & $\begin{array}{c}1.5259 \\
1.0640 \\
6.2367 \times 10^{-2} \\
\end{array}$ & $\begin{array}{l}4.6191 \times 10^{-1} \\
4.4252 \times 10^{-1} \\
3.1760 \times 10^{-2} \\
\end{array}$ \\
\hline & $\begin{array}{l}\lambda_{1}=10^{-6} \\
\lambda_{1}=10^{-3} \\
\lambda_{1}=10^{-1}\end{array}$ & $\lambda_{2}=0$ & $\begin{array}{l}\underline{0.6} \\
\underline{0.7} \\
\underline{0.6}\end{array}$ & $\begin{array}{c}1.1694 \\
9.7398 \times 10^{-1} \\
1.0836 \times 10^{1}\end{array}$ & $\begin{array}{l}4.8164 \\
3.2081 \\
4.4080\end{array}$ & $\begin{array}{l}1.6659 \\
1.0049 \\
1.9817\end{array}$ \\
\hline
\end{tabular}

Example 2. Consider a three-dimensional annular domain with an unknown inner boundary of radius $r^{(a n)}=\frac{1}{4}(1+\cos \theta \sin 2 \varphi)$. The boundary data are given as follows:

$$
\begin{aligned}
& \left.u\right|_{\partial \Omega}=f(\theta, \varphi)=3 \sin ^{2} \varphi-2 \\
& \left.\frac{\partial u}{\partial n}\right|_{\partial \Omega}=g(\theta, \varphi)=6 \sin ^{2} \varphi-4 \\
& \left.u\right|_{\partial D}=h(\theta, \varphi)=\frac{1}{16}\left(3 \sin ^{2} \varphi-2\right)(\sin 2 \varphi \cos \theta+1)^{2}
\end{aligned}
$$

The exact solution for these input boundary data is $u(x)=x_{1}^{2}+x_{2}^{2}-2 x_{3}^{2}$.

Table 4. The values of the minimal objective functions and the corresponding errors with initial guess

\begin{tabular}{|c|c|c|c|c|c|c|c|c|}
\hline $\mathbf{M}=\mathbf{N}$ & $\begin{array}{l}\text { With/No } \\
\text { Noise }\end{array}$ & $\begin{array}{l}\text { With/No } \\
\text { Regularisation }\end{array}$ & $\lambda_{1}$ & $\lambda_{2}$ & $r_{0}$ & $\begin{array}{l}\text { Objective } \\
\text { Function }\end{array}$ & $L_{\infty}$-Error & RMSE \\
\hline \multirow{2}{*}{25} & no & $\begin{array}{l}\text { no } \\
\text { with }\end{array}$ & 0 & $10^{-1}$ & $\underline{0.5}$ & $\begin{array}{l}4.9013 \times 10^{-3} \\
2.2118 \times 10^{-3}\end{array}$ & $\begin{array}{c}4.3884 \\
1.0442 \times 10^{-1}\end{array}$ & $\begin{array}{c}1.4418 \\
1.3354 \times 10^{-1}\end{array}$ \\
\hline & with & $\begin{array}{l}\text { no } \\
\text { with }\end{array}$ & 0 & $10^{-1}$ & $\underline{0.6}$ & $\begin{array}{c}2.1799 \\
1.2386 \times 10^{-2}\end{array}$ & $\begin{array}{c}2.1210 \\
1.0866 \times 10^{-1}\end{array}$ & $\begin{array}{l}7.1903 \times 10^{-1} \\
1.1611 \times 10^{-1}\end{array}$ \\
\hline \multirow[t]{2}{*}{50} & no & $\begin{array}{l}\text { no } \\
\text { with }\end{array}$ & 0 & $10^{-1}$ & $\underline{0.6}$ & $\begin{array}{c}6.1682 \\
1.6998 \times 10^{-5}\end{array}$ & $\begin{array}{c}5.0114 \\
9.8441 \times 10^{-3}\end{array}$ & $\begin{array}{c}1.7985 \\
1.1249 \times 10^{-2} \\
\end{array}$ \\
\hline & with & $\begin{array}{l}\text { no } \\
\text { with }\end{array}$ & 0 & $10^{-1}$ & $\frac{0.6}{0.6}$ & $\begin{array}{l}1.8326 \times 10^{1} \\
6.2499 \times 10^{-3}\end{array}$ & $\begin{array}{c}1.0113 \\
6.2367 \times 10^{-2}\end{array}$ & $\begin{array}{l}9.1243 \times 10^{-1} \\
3.1760 \times 10^{-2}\end{array}$ \\
\hline
\end{tabular}
$r_{0}$, obtained (with/no) selecting the optimal regularisation parameters with $M=N \in\{25,50\}$ for Example 1 .

Table 5 gives the values of the objective functions and the corresponding errors obtained using the optimal initial guess $r_{0}, M=N \in\{25,50\}$ without using regularisation parameters, whilst Tables 6 and 7 are obtained using the regularisation parameters $\lambda_{1}, \lambda_{2}$ and so, in Table 8 we give the minimal objective functions and the corresponding errors with initial guess $r_{0}$.

Table 5. The values of the optimal initial guess, $r_{0}$, objective functions and the corresponding errors for Example 2 with $M=N \in\{25,50\}$ and no regularisation parameters .

\begin{tabular}{cccccc}
\hline & $M=N$ & $r_{0}$ & Objective Functions & $L_{\infty}$-Error & RMSE \\
\hline \multirow{2}{*}{ Without noise } & 25 & $\underline{0.5}$ & $5.0884 \times 10^{1}$ & $4.1355 \times 10^{-1}$ & $6.0305 \times 10^{-1}$ \\
& 50 & $\underline{0.6}$ & $1.0837 \times 10^{2}$ & $8.1024 \times 10^{-1}$ & 1.2263 \\
\hline \multirow{2}{*}{ With noise 5\% } & 25 & $\underline{0.8}$ & $3.2489 \times 10^{1}$ & 1.9251 & 1.9305 \\
& 50 & $\underline{0.7}$ & $5.4166 \times 10^{1}$ & 4.5130 & 1.8355 \\
\hline
\end{tabular}


Table 6. The values of the optimal initial guess, $r_{0}$, objective functions and the corresponding errors using the regularisation parameters $\lambda_{1}, \lambda_{2} \in\left\{0,10^{-6}, 10^{-3}, 10^{-1}\right\}$ with $M=N=25$ for Example 2.

\begin{tabular}{|c|c|c|c|c|c|c|}
\hline & & & $r_{0}$ & Objective Functions & $L_{\infty}$-Error & RMSE \\
\hline \multirow{2}{*}{ 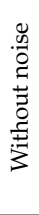 } & $\lambda_{1}=0$ & $\begin{array}{l}\lambda_{2}=10^{-6} \\
\lambda_{2}=10^{-3} \\
\lambda_{2}=10^{-1}\end{array}$ & $\begin{array}{l}\underline{0.5} \\
\underline{0.4} \\
\underline{0.8} \\
\end{array}$ & $\begin{array}{c}5.3972 \times 10^{1} \\
4.1825 \times 10^{1} \\
2.0433 \times 10^{-1} \\
\end{array}$ & $\begin{array}{l}5.0754 \times 10^{-1} \\
4.0134 \times 10^{-1} \\
4.0078 \times 10^{-1} \\
\end{array}$ & $\begin{array}{l}6.3467 \times 10^{-1} \\
4.7861 \times 10^{-1} \\
4.5618 \times 10^{-1} \\
\end{array}$ \\
\hline & $\begin{array}{l}\lambda_{1}=10^{-6} \\
\lambda_{1}=10^{-3} \\
\lambda_{1}=10^{-1}\end{array}$ & $\lambda_{2}=0$ & $\begin{array}{l}\underline{0.6} \\
\underline{0.1} \\
\underline{0.4}\end{array}$ & $\begin{array}{c}4.0430 \times 10^{1} \\
5.9128 \\
2.5867 \times 10^{1}\end{array}$ & $\begin{array}{c}1.5634 \\
4.9809 \times 10^{-1} \\
1.9664\end{array}$ & $\begin{array}{c}1.3413 \\
5.6395 \times 10^{-1} \\
2.9192\end{array}$ \\
\hline \multirow{2}{*}{ 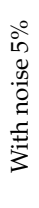 } & $\lambda_{1}=0$ & $\begin{array}{l}\lambda_{2}=10^{-6} \\
\lambda_{2}=10^{-3} \\
\lambda_{2}=10^{-1}\end{array}$ & $\begin{array}{l}\underline{0.8} \\
\underline{0.2} \\
\underline{0.1}\end{array}$ & $\begin{array}{c}3.3672 \times 10^{1} \\
5.4494 \\
1.9182 \times 10^{-1} \\
\end{array}$ & $\begin{array}{c}1.9251 \\
5.3183 \times 10^{-1} \\
4.3568 \times 10^{-1} \\
\end{array}$ & $\begin{array}{c}1.9396 \\
4.8568 \times 10^{-1} \\
4.7239 \times 10^{-1} \\
\end{array}$ \\
\hline & $\begin{array}{l}\lambda_{1}=10^{-6} \\
\lambda_{1}=10^{-3} \\
\lambda_{1}=10^{-1}\end{array}$ & $\lambda_{2}=0$ & $\begin{array}{l}\frac{0.8}{0.2} \\
\underline{0.4}\end{array}$ & $\begin{array}{c}3.4257 \\
5.9130 \\
2.5843 \times 10^{1}\end{array}$ & $\begin{array}{c}4.6055 \\
6.4875 \times 10^{-1} \\
1.9654\end{array}$ & $\begin{array}{c}2.1547 \\
6.2476 \times 10^{-1} \\
2.9119\end{array}$ \\
\hline
\end{tabular}

Table 7. The values of the optimal initial guess, $r_{0}$, objective functions and the corresponding errors using the regularisation parameters $\lambda_{1}, \lambda_{2} \in\left\{0,10^{-6}, 10^{-3}, 10^{-1}\right\}$ with $\mathrm{M}=\mathrm{N}=50$ for Example 2 .

\begin{tabular}{|c|c|c|c|c|c|c|}
\hline & & & $r_{0}$ & Objective Functions & $L_{\infty}$-Error & RMSE \\
\hline \multirow{6}{*}{ 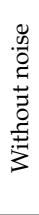 } & & $\lambda_{2}=10^{-6}$ & $\underline{0.4}$ & $8.3494 \times 10^{1}$ & $8.2159 \times 10^{-1}$ & 1.2749 \\
\hline & $\lambda_{1}=0$ & $\lambda_{2}=10^{-3}$ & $\underline{0.2}$ & $1.4682 \times 10^{-4}$ & $8.0200 \times 10^{-1}$ & 1.0192 \\
\hline & & $\lambda_{2}=10^{-1}$ & $\underline{0.8}$ & 3.8174 & $9.5145 \times 10^{-1}$ & 1.3288 \\
\hline & $\lambda_{1}=10^{-6}$ & & $\underline{0.5}$ & 2.0732 & 6.4408 & 2.9474 \\
\hline & $\lambda_{1}=10^{-3}$ & $\lambda_{2}=0$ & 0.7 & 3.6352 & 5.2101 & 3.879 \\
\hline & $\lambda_{1}=10^{-1}$ & & $\underline{0.4}$ & $4.7401 \times 10^{1}$ & $1.1726 \times 10^{1}$ & 7.6963 \\
\hline \multirow{6}{*}{ 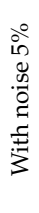 } & & $\lambda_{2}=10^{-6}$ & $\underline{0.8}$ & $3.6080 \times 10^{1}$ & $8.5674 \times 10^{-1}$ & 1.1744 \\
\hline & $\lambda_{1}=0$ & $\lambda_{2}=10^{-3}$ & $\overline{0.9}$ & $2.1617 \times 10^{-1}$ & $5.2427 \times 10^{-1}$ & $6.5482 \times 10^{-1}$ \\
\hline & & $\lambda_{2}=10^{-1}$ & $\overline{0.7}$ & $3.2234 \times 10^{-2}$ & $4.7546 \times 10^{-1}$ & $4.9726 \times 10^{-1}$ \\
\hline & $\lambda_{1}=10^{-6}$ & & $\underline{0.6}$ & $1.0459 \times 10^{1}$ & $6.7322 \times 10^{-1}$ & $6.447 \times 10^{-1}$ \\
\hline & $\lambda_{1}=10^{-3}$ & $\lambda_{2}=0$ & $\underline{0.6}$ & 7.0388 & $9.6421 \times 10^{-1}$ & 1.1551 \\
\hline & $\lambda_{1}=10^{-1}$ & & $\overline{0.7}$ & $7.5548 \times 10^{1}$ & $3.4477 \times 10^{3}$ & $1.2448 \times 10^{3}$ \\
\hline
\end{tabular}

Table 8. The values of the minimal objective functions and the corresponding errors with initial guess, $r_{0}$, obtained (with/no) selecting the optimal regularisation parameters $\lambda_{1}, \lambda_{2}$ with $M=N \in\{25,50\}$ for Example 2.

\begin{tabular}{|c|c|c|c|c|c|c|c|c|}
\hline $\mathbf{M}=\mathbf{N}$ & $\begin{array}{l}\text { With/No } \\
\text { Noise }\end{array}$ & $\begin{array}{l}\text { With/No } \\
\text { Regularisation }\end{array}$ & $\lambda_{1}$ & $\lambda_{2}$ & $r_{0}$ & $\begin{array}{l}\text { Objective } \\
\text { Function }\end{array}$ & $L_{\infty}$-Error & RMSE \\
\hline \multirow{2}{*}{25} & no & $\begin{array}{l}\text { no } \\
\text { with }\end{array}$ & 0 & $10^{-1}$ & $\underline{0.5}$ & $\begin{array}{l}.0884 \times 10^{1} \\
2.0433 \times 10^{-1}\end{array}$ & $\begin{array}{l}4.1355 \times 10^{-1} \\
4.0078 \times 10^{-1}\end{array}$ & $\begin{array}{l}6.0305 \times 10^{-1} \\
4.5618 \times 10^{-1}\end{array}$ \\
\hline & with & $\begin{array}{l}\text { no } \\
\text { with }\end{array}$ & 0 & $10^{-1}$ & $\underline{0.8}$ & $\begin{array}{l}3.2489 \times 10^{1} \\
1.9182 \times 10^{-1}\end{array}$ & $\begin{array}{c}1.9251 \\
4.3568 \times 10^{-1}\end{array}$ & $\begin{array}{c}1.9305 \\
4.7239 \times 10^{-1}\end{array}$ \\
\hline \multirow{2}{*}{50} & no & $\begin{array}{l}\text { no } \\
\text { with }\end{array}$ & 0 & $10^{-3}$ & $\underline{0.6}$ & $\begin{array}{l}1.0837 \times 10^{2} \\
1.4682 \times 10^{-4}\end{array}$ & $\begin{array}{l}8.1024 \times 10^{-1} \\
8.0200 \times 10^{-1}\end{array}$ & $\begin{array}{l}1.2263 \\
1.0192 \\
\end{array}$ \\
\hline & with & $\begin{array}{l}\text { no } \\
\text { with }\end{array}$ & 0 & $10^{-1}$ & $\frac{0.7}{0.7}$ & $\begin{array}{l}5.4166 \times 10^{1} \\
3.2234 \times 10^{-2}\end{array}$ & $\begin{array}{c}4.5130 \\
4.7546 \times 10^{-1}\end{array}$ & $\begin{array}{c}1.8355 \\
4.9726 \times 10^{-1}\end{array}$ \\
\hline
\end{tabular}

\section{Conclusions}

In this paper, we extended the aforementioned method presented in [8], based on the method of fundamental solutions to solve numerically the three-dimensional inverse geometry problem on an annular domain. To obtain the stable and accuracy results, Tikhonov's regularisation parameters were used combined with the problem of the minimising an objective function. From the examples, we can see that our proposed method is effective and stable, even for the boundary data added with noise.

Author Contributions: Validation, H.A.; formal analysis, E.A., C.P.; investigation, M.S., H.A., E.A., C.P.; writing—original draft preparation, M.S.; writing—review and editing, H.A.; supervision, M.S.; funding acquisition, C.P. All authors have read and agreed to the published version of the manuscript. 
Funding: This work does not receive any external funding.

Institutional Review Board Statement: Not applicable.

Informed Consent Statement: Not applicable.

Data Availability Statement: Data sharing is not applicable to this article, as no data set was generated or analysed during the current study.

Conflicts of Interest: The authors declare no confilct of interests.

\section{References}

1. Lesnic, D.; Berger, J.R.; Martin, P.A. A boundary element regularization method for the boundary determination in potential corrosion damage. Inverse Probl. Eng. 2002, 10, 163-182. [CrossRef]

2. Karageorghis, A.; Lesnic, D.; Marin, L. The method of fundamental solutions for the detection of rigid inclusions and cavities in plane linear elastic bodies. Comput. Struct. 2012, 106, 176-188. [CrossRef]

3. Karageorghis, A.; Lesnic, D.; Marin, L. A moving pseudo-boundary method of fundamental solutions for void detection. Numer. Methods Partial Diff. Equat. 2013, 29, 935-960. [CrossRef]

4. Karageorghis, A.; Lesnic, D.; Marin, L. The method of fundamental solutions for three-dimensional inverse geometric elasticity problems. Comput. Struct. 2016, 166, 51-59. [CrossRef]

5. Wanga, F.; Huaa, Q.; Liub, C.S. Boundary function method for inverse geometry problem in two-dimensional anisotropic heat conduction equation. Appl. Math. Lett. 2018, 84, 130-136. [CrossRef]

6. Huang, C.H.; Yan, J.Y. An inverse problem in simultaneously measuring temperature dependent thermal conductivity and heat capacity. Int. J. Heat Mass Transf. 1995, 38, 3433-3441. [CrossRef]

7. Huang, C.H.; Yeh, C.Y.; Orlande, H.R.B. A non-linear inverse problem in simultaneously estimating the heat and mass production rates for a chemically reacting fluid. Chem. Eng. Sci. 2003, 58, 3741-3752. [CrossRef]

8. Bin-Mohsin, B.; Lesnic, D. Determination of inner boundaries in modified Helmholtz inverse geometric problems using the method of fundamental solutions. Math. Comput. Simul. 2012, 82, 1445-1458. [CrossRef]

9. Vladimirov, V.S. Equations of Mathematical Physics; Mir Publishers: Moscow, Russia, 1981. 